\title{
Management of Compound Metatarsal Fractures under Tamil Nadu Accident and Emergency Care Initiative
}

\author{
Vetrivel Chezian Sengodan ${ }^{1}$, Arun Subramanian ${ }^{2}$
}

\begin{abstract}
Introduction: Metatarsal fractures are common among foot injuries. This study aimed to evaluate and analyze the functional outcomes of compound metatarsal fractures using the anterograde retrograde fixation technique under the Tamil Nadu Accident and Emergency Care Initiative. Materials and methods: This is a prospective study done on patients admitted with compound metatarsal fractures in emergency orthopedic unit, Government Coimbatore Medical Hospital under Tamil Nadu Accident and Emergency Care Initiative between January 2020 and December 2020. The displaced compound metatarsal fractures are treated with limited internal fixation using Kirschner's wires within the golden period of 6 hours and they were covered under Government of Tamil Nadu Chief Minister's Comprehensive Health Insurance Scheme-Ayushman Bharat Pradhan Mantri Jan Arogya Yojana (TNCMCHIS-AB-PMJAY). Anterograde retrograde technique was used for the fixation of compound metatarsal fractures.

Results: The American Orthopaedic Foot and Ankle Society score was used to assess the functional outcome of compound metatarsal fractures. Good results were observed in 32 patients and excellent results were observed in 5 patients. Fair results were seen in three patients.

Conclusion: The compound undisplaced metatarsal fractures can be treated conservatively. The displaced metatarsal fractures need limited internal fixation with Kirschner's wires. The Tamil Nadu accident and emergency care initiative provide early treatment and early discharge of the patients. The health department is no more an expenditure department for the government since it generates money from TNCMCHISAB-PMJAY which is very useful for the patients from below poverty line.

Keywords: Government of Tamil Nadu chief minister's comprehensive health insurance scheme, Kirschner wire, Metatarsal fractures, Tamil Nadu accident and emergency care initiative.

TNOA Journal of Orthopedics and Joint Surgery (2021): 10.5005/jp-journals-10079-1047
\end{abstract}

\section{INTRODUCTION}

Metatarsal fractures are common among foot injuries. Middle-age people are most commonly injured. One or all the metatarsals may be injured which may be a complete or incomplete fracture. Metatarsals are placed in the foot between the toes and the tarsal bones. Forefoot fractures may be due to direct or indirect injury. The most common mode of injury is road traffic accidents which are always high-velocity injuries. It can be a closed fracture or a compound fracture (Fig. 1). If left untreated, they usually produce pain and limitations in activities of daily living. Metatarsal injuries can also involve the Lisfranc joint. Anatomically, they are classified into proximal metaphyseal (base), diaphyseal (shaft), cervical (neck), and cephalic (head) (Fig. 2). Metatarsal fractures that are not displaced are managed by conservative treatment. Fractures that are minimally displaced in the frontal plane, with no shortening or angulation, can be treated conservatively. Compound fractures, compartment syndrome, and unreduced joint dislocations are treated surgically. Complications of compound forefoot injuries are metatarsalgia which may be due to malunion of these fractures. The reduction and stability of all metatarsal fractures cannot be achieved satisfactorily by a closed reduction maneuver. This led to the development of several surgical techniques. ${ }^{1-7}$ Limited internal fixation with Kirschner's wires is widely used to fix these fractures. If it is a closed fracture, then the fractures can be fixed percutaneously but if the fractures are found to be compound, then it has to be reduced and fixed by open surgical technique after wound debridement. Compound fractures are usually fixed using the "antegrade retrograde technique". This study aimed to evaluate

\begin{abstract}
${ }^{1,2}$ Institute of Orthopaedics and Traumatology, Coimbatore Medical College and Hospital, Coimbatore, Tamil Nadu, India

Corresponding Author: Vetrivel Chezian Sengodan, Institute of Orthopaedics and Traumatology, Coimbatore Medical College and Hospital, Coimbatore, Tamil Nadu, India, Phone: +91 9843028096, e-mail: svcortho@gmail.com
\end{abstract}

How to cite this article: Sengodan VC, Subramanian A. Management of Compound Metatarsal Fractures under Tamil Nadu Accident and Emergency Care Initiative. TNOA J Orth Joint Surg 2021;3(2):70-75.

Source of support: Nil

Conflict of interest: None

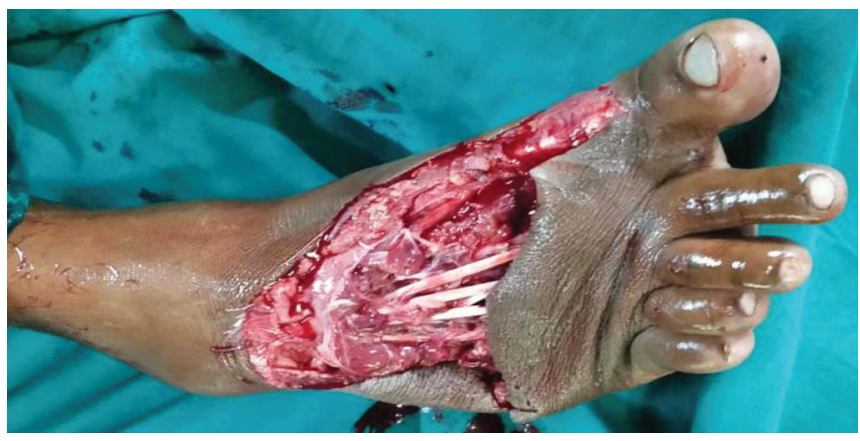

Fig. 1: Compound foot injury

(c) The Author(s). 2021 Open Access This article is distributed under the terms of the Creative Commons Attribution 4.0 International License (https:// creativecommons.org/licenses/by-nc/4.0/), which permits unrestricted use, distribution, and non-commercial reproduction in any medium, provided you give appropriate credit to the original author(s) and the source, provide a link to the Creative Commons license, and indicate if changes were made. The Creative Commons Public Domain Dedication waiver (http://creativecommons.org/publicdomain/zero/1.0/) applies to the data made available in this article, unless otherwise stated. 
and analyze the functional outcomes of compound metatarsal fractures using the anterograde retrograde fixation technique under the Tamil Nadu Accident and Emergency Care Initiative.

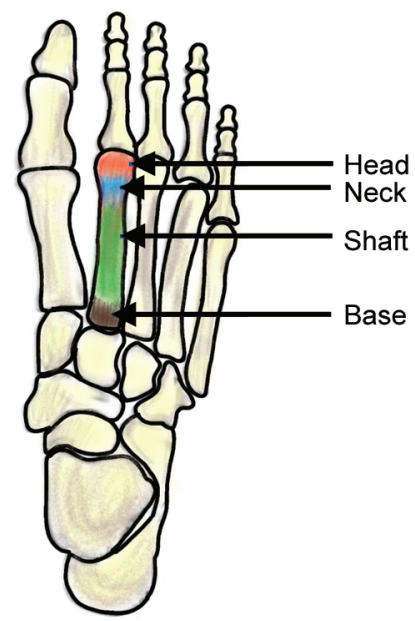

Fig. 2: Anatomy of metatarsal

\section{Materials and Methods}

This study was done between January 2020 and December 2020. The study was done at the Institute of Orthopaedics and Traumatology, Government Coimbatore Medical College Hospital after ethical committee clearance. The "Tamil Nadu accident and emergency care initiative" program was implemented by the Government of Tamil Nadu in 2016. With the implementation of the Tamil Nadu accident and emergency care initiative program, leg bone fractures, distal radius fractures, compound fractures including compound metatarsal fractures are fixed within the golden period of 6 hours. Government Coimbatore Medical College Hospital is a tertiary care center. It serves the people below the poverty line not only from Coimbatore but also from the neighboring districts namely Tiruppur, Nilgiris, Erode, and neighboring states namely Kerala and Karnataka. The Government provides the treatment free of cost. A separate orthopedic emergency operation theater, facilities like $C$ arm, orthopedic implants and instruments, autoclave machine is available round the clock for performing emergency orthopedic surgical procedures (Fig. 3). Qualified orthopedic surgeons, theater staffs are available round the clock for effective healthcare delivery and betterment of the patient.
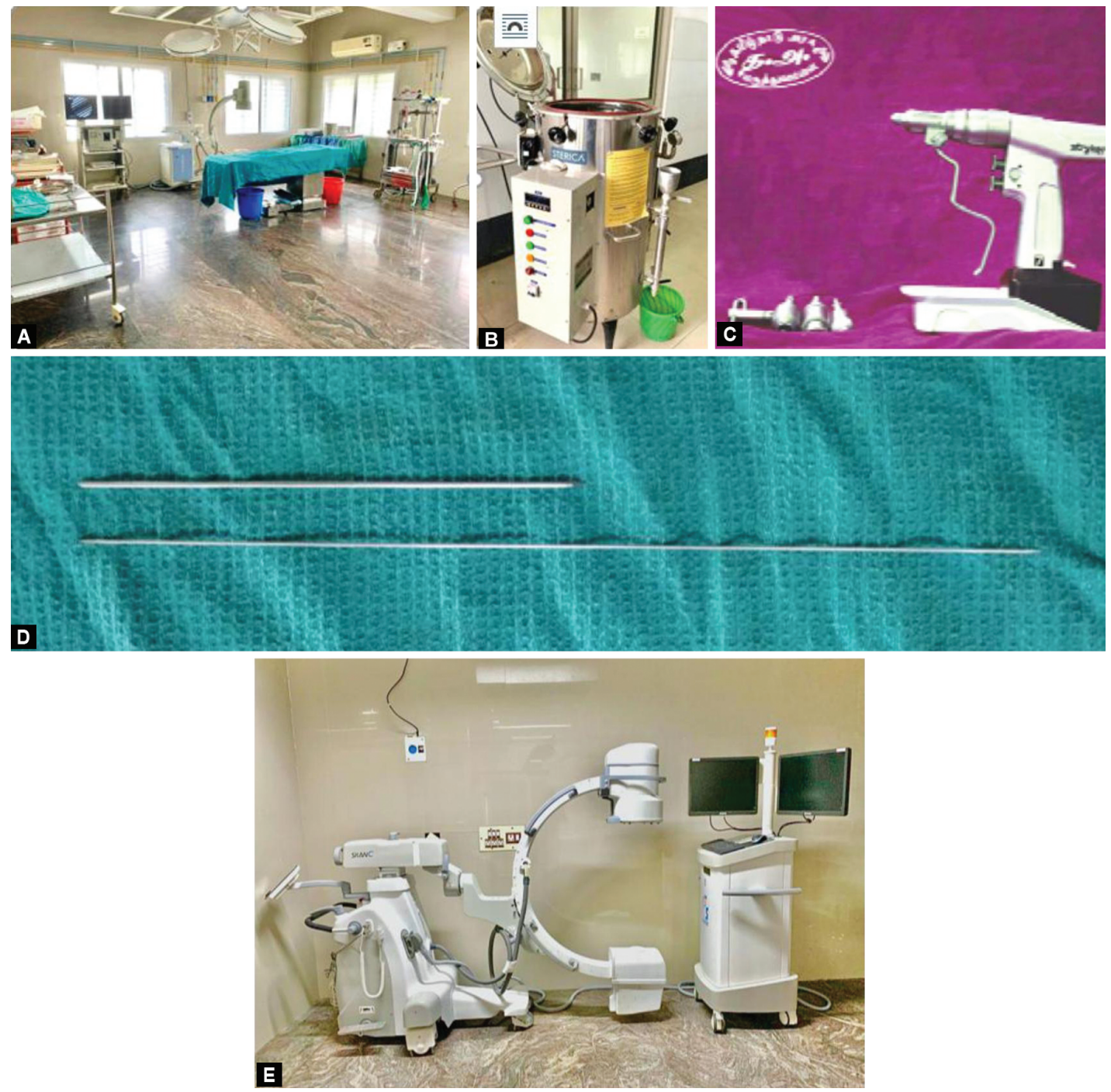

Figs 3A to E: (A) Emergency operation theater; (B) Autoclave machine; (C) Drill machine; (D) Kirschner wires; (E) C-arm machine 
A total of 46 patients were in the study group. Only compound metatarsal fractures were included in our study, closed fractures and pediatric fractures were excluded from our study. Preoperatively, 2 $\mathrm{g}$ of injection ceftriaxone was given to control infection after wound swab for culture and sensitivity and the appropriate antibiotic was continued postoperatively.

The hematological and radiological investigation results will be available within one hour. The fracture pattern will be studied from the radiographs. The undisplaced fractures were treated conservatively and the displaced fractures were treated surgically with Kirschner wires.

Kirschner's wire which is a limited internal fixation device is most commonly used for fixation of metatarsal fractures. $1.5-2 \mathrm{~mm}$ Kirschner's wires were used for the fixation of metatarsal fractures.

The American Orthopaedic Foot and Ankle Society score was used to evaluate the outcome and it consists of seven questions on pain, activity and functional limitations, footwear, walking distance, difficulties with different terrains, gait, and alignment (whether or not plantigrade). The seven questions related to three components: Pain (1 question; maximum 40 points), function (5 questions; maximum 45 points), and alignment (1 question; maximum 15 points) leading to a total possible score of 100 points (Table 1).

\section{Procedure}

In our study, we used Kirschner's wires to fix the compound metatarsal fractures. "Anterograde and retrograde technique" was used to fix these fractures. In this technique, Kirschner's wires were inserted through the distal fragment and brought out through the sole. The wires are then grabbed by the drill at the sole and drilled back such that the tip of the wire is at the fracture site. Now the fracture is reduced and checked with the C-arm and the wire is advanced through the fracture site (Fig. 4). The reduction and position of Kirschner's wire are confirmed using the C-arm. This technique is simple, less time-consuming, and radiation exposure is also minimal. The metatarsal fracture fixation by Kirschner's wires
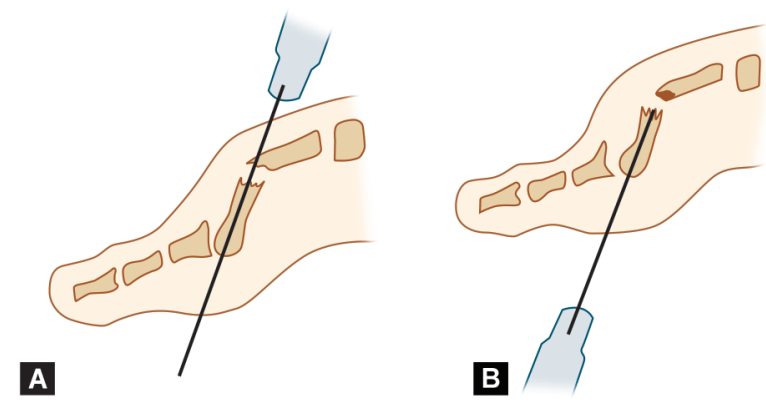

c
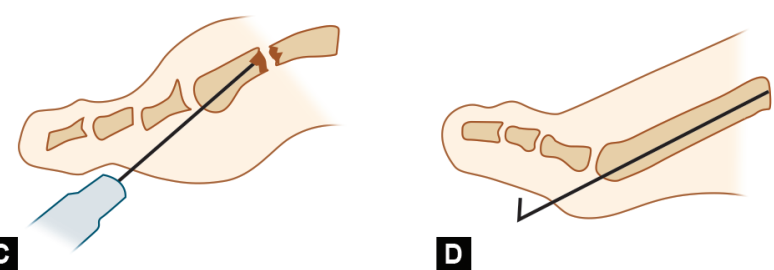

Figs 4A to D: (A) Kirschner's wires inserted through the distal fragment and brought out through the sole of the foot; (B) Kirschner wire is then grabbed by the drill at the sole of the foot; (C) Fracture is reduced; (D) Wire is advanced through the fracture site will provide only limited internal fixation and it will not provide rotational stability.

\section{Postoperative Protocol}

Immediately after the operation, a below-knee plaster is applied for 4 weeks to prevent further displacements. In our study, weightbearing is allowed 4-6 weeks after the procedure depending on the radiological follow-up. In the meantime, the toes were mobilized to prevent stiffness. Removal of $\mathrm{K}$ wires was done around 6 weeks after surgery.

\section{Results}

In our study, 41 patients were males, and the remaining 5 patients were females (Fig. 5). The mode of injury was found to be road traffic

Table 1: The American Orthopaedic Foot and Ankle Society score

\begin{tabular}{|c|c|}
\hline \multicolumn{2}{|l|}{ American Orthopaedic Foot and Ankle Society score } \\
\hline \multicolumn{2}{|l|}{ Pain (40 points) } \\
\hline None & 40 \\
\hline Mild, occasional & 30 \\
\hline Moderate, daily & 20 \\
\hline Severe, almost always present & 0 \\
\hline \multicolumn{2}{|l|}{ Function (45 points) } \\
\hline \multicolumn{2}{|l|}{ Activity limitation, support } \\
\hline No limitation, no support & 10 \\
\hline $\begin{array}{l}\text { No limitation of daily activities, limitation of } \\
\text { recreational activities, no support }\end{array}$ & 7 \\
\hline Limited daily and recreational activities, cane & 4 \\
\hline $\begin{array}{l}\text { Severe limitation of daily and recreational activities, } \\
\text { walker, crutches, wheeler }\end{array}$ & 0 \\
\hline \multicolumn{2}{|l|}{ Maximum walking distance, blocks } \\
\hline$>6$ & 5 \\
\hline $4-6$ & 4 \\
\hline $1-3$ & 2 \\
\hline$<1$ & 0 \\
\hline \multicolumn{2}{|l|}{ Footwear requirements } \\
\hline Fashionable, conventional shoes, no insert required & 5 \\
\hline Comfort footwear, shoe insert & 3 \\
\hline Modified shoes/brace & 0 \\
\hline \multicolumn{2}{|l|}{ Walking surfaces } \\
\hline No difficulty on any surface & 10 \\
\hline $\begin{array}{l}\text { Some difficulty on uneven terrain, stairs, inclines, } \\
\text { ladders }\end{array}$ & 5 \\
\hline $\begin{array}{l}\text { Severe difficulty on uneven terrain, stairs, inclines, } \\
\text { ladders }\end{array}$ & 0 \\
\hline \multicolumn{2}{|l|}{ Gait abnormality } \\
\hline None, slight & 10 \\
\hline Obvious & 5 \\
\hline Marked & 0 \\
\hline \multicolumn{2}{|l|}{ Alignment (15 points) } \\
\hline Good, plantigrade foot, midfoot well aligned & 10 \\
\hline $\begin{array}{l}\text { Fair, plantigrade foot, some degree of midfoot } \\
\text { malalignment observed, no symptoms }\end{array}$ & 8 \\
\hline $\begin{array}{l}\text { Poor, non-plantigrade foot, severe malalignment, } \\
\text { symptoms }\end{array}$ & 0 \\
\hline Total & 100 \\
\hline
\end{tabular}




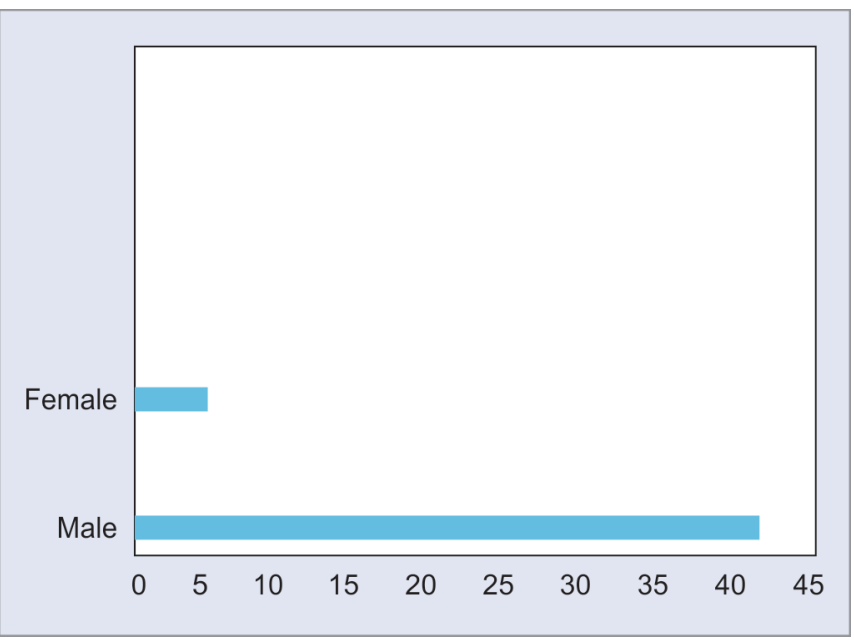

Fig. 5: Sex pattern of compound metatarsal fractures in our study

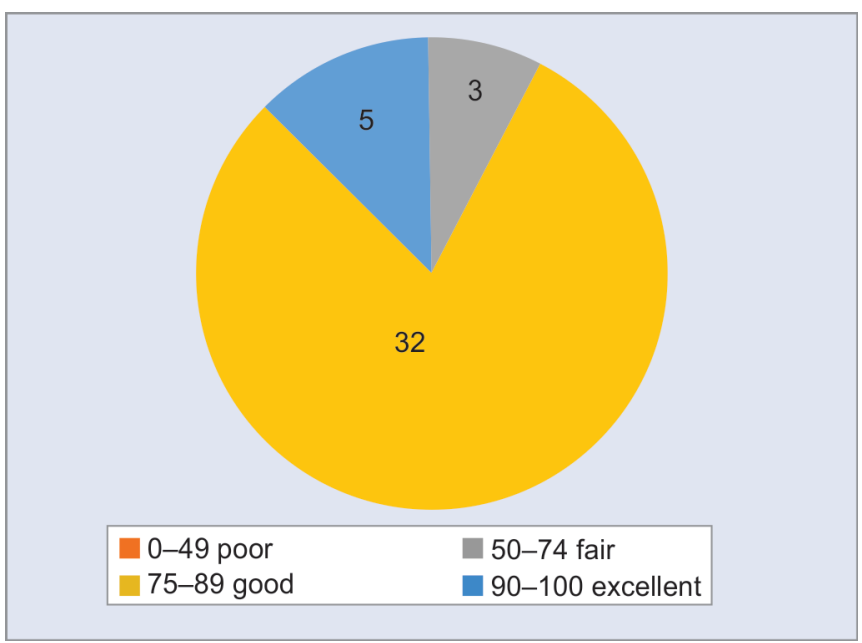

Fig. 7: AOFAS score-result interpretation

accident in 41 cases, accidental fall in 3 cases and workspot injury in 2 cases (Fig. 6). The average duration of surgery was $<1$ hour. Intraoperatively, no significant complications were noted. Mean period of stay in the hospital was 5 days.

The average time for bony union is found to be 2 months except for few cases with extensive soft tissue damage.

In our study, the mean time for full weight-bearing was 7.4 weeks. Kirschner wires were removed at the period of 6-10 weeks after confirming the bony union using serial X-rays. In our study, the bony union was achieved in 40 patients and 6 patients had lost follow-up. Five patients had superficial infection and were treated with systemic antibiotics. In our study, in two cases, there was deep infection. Wound debridement was done, wound swab was sent for culture and sensitivity and managed with appropriate antibiotics. In our study, loss of reduction were noted in two patients. Metatarsalgia was noted in four cases. Four patients had stiffness over the metatarsophalangeal joints and was treated with physiotherapy.

If the AOFAS score is between 0 and 49, then the outcome is poor. The outcome is fair when the score is between 50 and 74 . When the score is between 75 and 89 , then the result is good. The result is excellent if the score is between 90 and 100 .

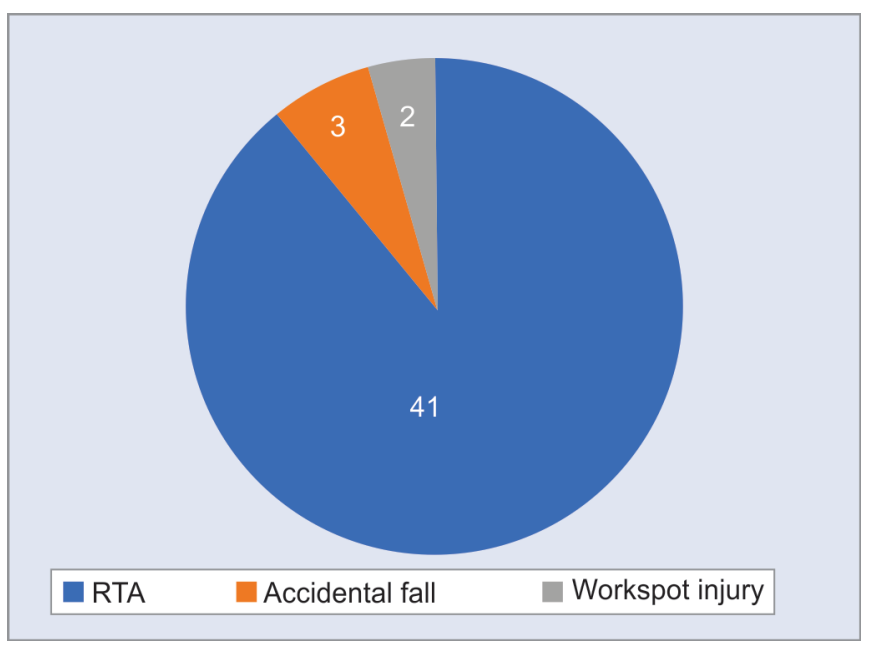

Fig. 6: Mode of injury

In our study, good results were observed in 32 patients and excellent results were observed in 5 patients. Fair results were seen in three patients. No patients had poor outcomes (Fig. 7).

In our study, the mean American Orthopaedic Foot and Ankle Society score was 85.68 .

\section{Discussion}

Tamil Nadu accident and emergency care initiative (TAEI) has six pillars (Fig. 8). One of the six pillars is Trauma. The six pillars are Stroke, ST-elevation Myocardial Infarction (STEMI), Trauma, Burns, Poisoning, Paediatric Resuscitation Emergency Management (PREM).

Most of the orthopedic procedures including metatarsal fractures are covered under of Government of Tamil Nadu Chief Minister's Comprehensive Health Insurance Scheme Ayushman Bharat Pradhan Mantri Jan Arogya Yojana (TNCMCHIS-AB-PMJAY) (Fig. 9). Hence money is generated by treating the patients below poverty line in the Government hospital. In our study, all the surgical fixation of metatarsal fractures were done under TNCMCHIS-ABPMJAY.

The advantages of TAEI are early treatment, decreasing the hospital stay, reduction of hospital expenditure, generating money from the insurance. After TAEl implementation, it helped the Tamil Nadu Government not only in maintaining its quality health care but also financially by treating the patients under TNCMCHIS-AB-PMJAY thereby generating more fund which can be used for improving the infrastructure, drugs, consumables and purchasing state of art equipment and instruments for the patient health care. ${ }^{8}$ Metatarsal fractures represent $3-7 \%$ of all fractures of the body and $35 \%$ of fractures of the foot. Globally, the compound metatarsal fractures were stabilized with limited internal fixation with Kirschner's wires. Fixation of metatarsal fractures in the antegrade technique needs pre bending of the Kirschner wire whereas in our study we did anterograde retrograde technique in which pre bending of Kirschner wire is not necessary.

Kim et al. from Korea studied 35 patients with matatarsal fractures fixed with intramedullary pinning and concluded that 33 patients had good functional outcome and 2 patients had stiffness. ${ }^{9}$

Zarei et al. from Iran concluded that, out of 34 patients who sustained metatarsal fractures and treated with limited internal 


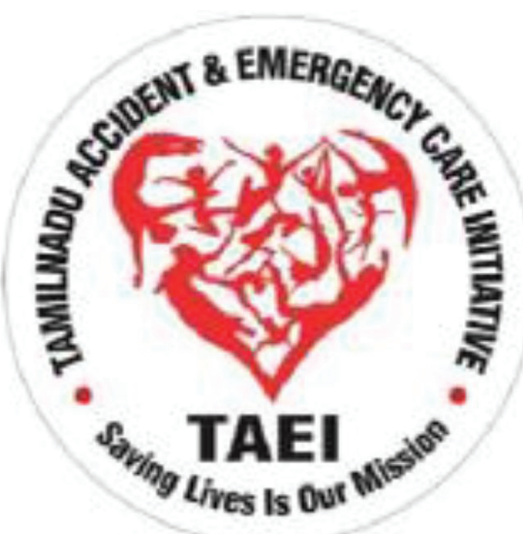

Fig. 8: Tamil Nadu Accident and Emergency Care Initiative

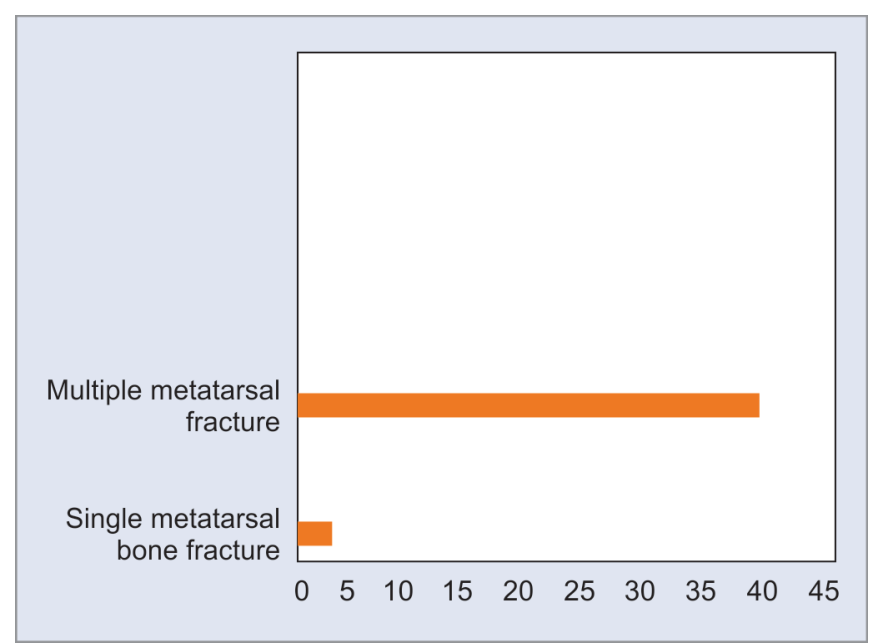

Fig. 10: Involvement of metatarsal fractures in our study

fixation, all achieved bony union and surgical site infection rate was nil in his study. ${ }^{10}$

Baumfeld et al. from Brazil observed that, among 14 patients with matatarsal fractures surgically treated by Kirschner's wires, bony union is achieved in all 14 patients and stiffness, metatarsalgia were not noted in any patients. ${ }^{11}$

Goyal et al. in India studied 10 patients with metatarsal fractures surgically treated with Kirschner's wires. All patients achieved good results in terms of walking, gait, and bone union. ${ }^{12}$

Kaushik et al. from India studied 50 cases of compound metatarsal fractures. More complications such as infection, malunion, deformity were noted in conservatively treated patients. Surgically treated patients achieved good bony union but the infection rate was higher. ${ }^{13}$

Goel et al. from India concluded that all seven patients with metatarsal neck fractures managed with Kirschner's wires achieved bony union in 8 weeks. ${ }^{14}$

Gade et al. from India studied 10 cases of metatarsal fractures. Nine cases of compound metatarsal fractures treated with Kirschner's wires achieved good bony union and had normal walking at the end of follow-up. One case had minimal complication. ${ }^{15}$

Bhat et al. from India studied 20 cases of metatarsal fractures. He concluded that anatomical reduction was obtained in 19
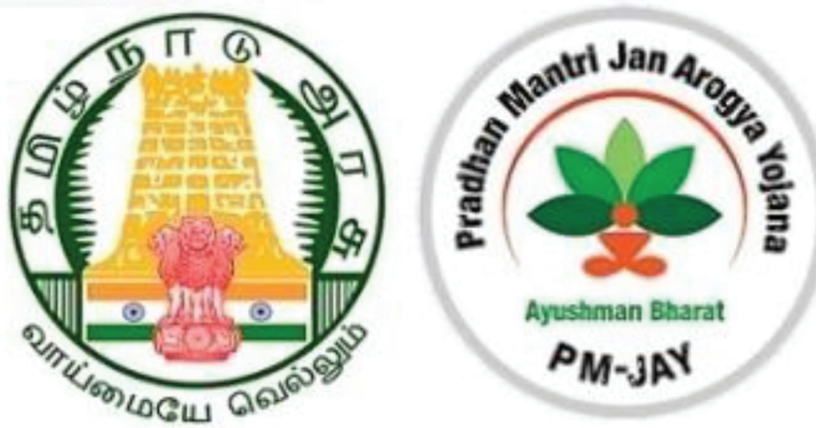

Fig. 9: Logo of Government of Tamil Nadu Chief Minister's Comprehensive Health Insurance Scheme-Ayushman Bharat Pradhan Mantri Jan Arogya Yojana (TNCMCHIS-AB-PMJAY)

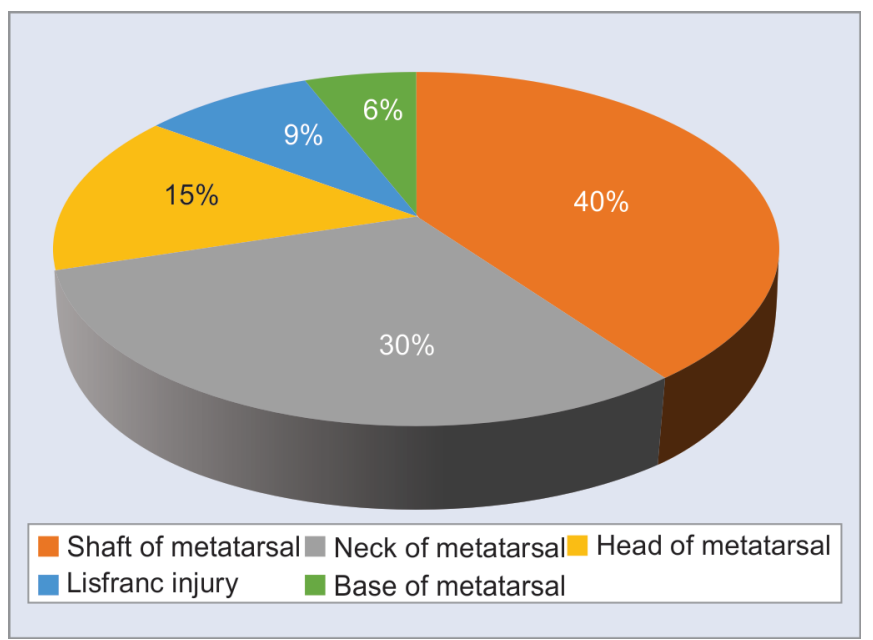

Fig. 11: Anatomical location of metatarsal fractures in our study

patients and 1 patient had loss of reduction. Good to fair results were obtained in $90 \%$ of cases in final follow-up. ${ }^{16}$

In our study, only 3 cases had isolated metatarsal bone fracture and the remaining 43 cases had more than one metatarsal bone fracture (Fig. 10). Isolated foot injuries were found in 35 cases and trauma involving other long bones or visceral injury was found in 11 cases. In our study, metatarsal fractures are further divided according to the site of fracture. In our study, fracture in the shaft of the metatarsal is more common (19 patients) followed by neck (14 patients), head (7 patients), and base (2 patients) of metatarsal in order. Lisfranc injury was found in four cases (Fig. 11).

Sheriff has given the following radiological criteria for surgical fixation of metatarsal fractures. ${ }^{17}$

- Fractures with $>3-\mathrm{mm}$ deviation.

- Fractures with $>10^{\circ}$ of angulation are surgically treated.

In our study, five cases of compound metatarsal fractures without displacement were managed with below-knee plasters and they achieved good bone union. In our study, 41 patients who sustained an unstable base fracture of the second metatarsal, multiple adjacent metatarsal fractures, severely comminuted fractures, hyperextended neck fractures were treated with surgical fixation after wound debridement. If 
managed well, the prognosis is good and the patients have low complication rates.

\section{Conclusion}

Metatarsal fracture is a common forefoot injury. The compound undisplaced metatarsal fractures can be treated conservatively. The displaced metatarsal fracture needs limited internal fixation with Kirschner's wires. In India, the healthcare delivery system in Tamil Nadu is always a role model for other states. The Tamil Nadu accident and emergency care initiative model can be used all over India to provide early treatment for the patients below the poverty line.

\section{References}

1. Mereddy PK, Molloy A, Hennessy MS. Osteochondral fracture of the fourth metatarsal head treated by open reduction and internal fixation. J Foot Ankle Surg 2007;46(4):320-322. DOI: 10.1053/j. jfas.2007.02.003.

2. Ozer $\mathrm{H}, \mathrm{Ozner} \mathrm{A}$. The transverse dorsal approach to displaced multimetatarsal fractures. J Foot Ankle Surg 2006;45(3):190-191. DOI: 10.1053/j.jfas.2006.02.009.

3. Peled E, Norman D, Melamed E. Metatarsal fracture reduction and fixation by Kirschner wires: technical tip. Surg Sci 2012;3(06):344-346. DOI: 10.4236/ss.2012.36067.

4. Donahue MP, Manoli A. Technical tip: transverse percutaneous pinning of metatarsal neck fractures. Foot Ankle Int 2004;25(6): 438-439. DOI: 10.1177/107110070402500615.

5. Early JS. Fractures and dislocations of the midfoot and forefoot. In: Fractures in adults, ed. Bucholz RW, Heckman JD, Court-Brown DM 6th ed., Philadelphia: Lippincott Williams and Wilkins; 2006. pp. 2370-2383.

6. Rammelt $\mathrm{S}$, Heineck J, Zwipp H. Metatarsal fractures. Injury 2004;35(suppl 2):SB77-SB86. DOI: 10.1016/j.injury.2004.07.016.

7. Aguado HJ, Herranz PG, Rapariz JM. Metaizeau's technique for displaced metatarsal neck fractures. J Pediatr Orthop 2004;12B:350-353.
8. Sengodan VC, Sivagnanam M, Kandasamy K. Impact of Tamil Nadu accident and emergency care initiative (TAEI) programme on orthopaedic emergency fixation: lesson from Coimbatore for low income countries. Int J Orthop Sci 2019;6(1):480-486. DOI: 10.22271/ ortho.2020.v6.i1i.1911.

9. Kim HN, Park YJ, Kim GL, et al. Closed antegrade intramedullary pinning for reduction and fixation of metatarsal fractures. J Foot Ankle Surg 2012;51(4):445-449. DOI: 10.1053/j.jfas.2012. 04.009.

10. Zarei M, Bagheri N, Nili A, et al. Closed antegrade/retrograde intramedullary fixation of central metatarsal fractures: surgical technique and clinical outcomes. Injury 2020;51(4):1125-1129. DOI: 10.1016/j.injury.2020.03.001.

11. Baumfeld D, Macedo BD, Nery C, et al. Anterograde percutaneous treatment of lesser metatarsal fractures: technical description and clinical results. RevistaBrasileira de Ortopedia (English Edition) 2012;47(6):760-764. DOI: 10.1016/S2255-4971(15)30035-5.

12. Goyal NB, Patil AV, Nishandar VV. Multiple metatarsal fracture fixation by k-wire: retrograde technique. Global J Res Analy 2018;7(8): 2277-8160.

13. Kaushik S, Verma RK, Gupta SP, et al. Evaluation of efficacy of different modalities in treatment of fracture metatarsal. Asian J Pharmaceut Clin Res 2021;14(5):974-2441.

14. Goel NK, Khurana A, Narula V, et al. Closed transverse pinning for reduction and fixation of metatarsal neck fractures: surgical technique. Indian J Orthop 2020;55(3):758-762. DOI: 10.1007/s43465020-00170-y.

15. Gade SS, Bhor P, Vatkar AJ, et al. VAC (cacuum assisted closure) therapy with $\mathrm{K}$ wire fixation as management protocol in complex compound foot fractures with soft tissue defects. Int J Orthop Sci 2019;5(3):205-208. DOI: 10.22271/ortho.2019.v5.i3d. 1532.

16. Bhat SA, Kangoo KA, Zahoor A, et al. Outcome after fluoroscopic guided closed reduction and percutaneous kirschner wire fixation of lisfranc joint injuries: a nationwide randomized controlled trial. Surgery Curr Res 2015;5(1):220.

17. Shereff MJ. Fractures of the forefoot. Instr Course Lect 1990;39: 133-140. 